\title{
Multi-modal tissue sampling in cone beam CT guided navigation bronchoscopy: comparative accuracy of different sampling tools and rapid on-site evaluation of cytopathology
}

\author{
Roel L. J. Verhoeven ${ }^{1 \wedge}$, Shoko $\operatorname{Vos}^{2}{ }^{\wedge}$, Erik H. F. M. van der Heijden ${ }^{1 \wedge}$ \\ ${ }^{1}$ Department of Pulmonology, Radboud University Medical Center, Nijmegen, The Netherlands; ${ }^{2}$ Department of Pathology, Radboud University \\ Medical Center, Nijmegen, The Netherlands \\ Contributions: (I) Conception and design: RLJ Verhoeven, EHFM van der Heijden; (II) Administrative support: All authors; (III) Provision of \\ study materials or patients: All authors; (IV) Collection and assembly of data: RLJ Verhoeven, EHFM van der Heijden; (V) Data analysis and \\ interpretation: All authors; (VI) Manuscript writing: All authors; (VII) Final approval of manuscript: All authors. \\ Correspondence to: Erik H. F. M. van der Heijden, MD, PhD. Associate Professor Interventional Pulmonology, Radboud University Nijmegen Medical \\ Centre, Department of Pulmonary Diseases, PO-Box 9101, 6500 HB Nijmegen, The Netherlands. Email: Erik.vanderHeijden@radboudumc.nl.
}

\begin{abstract}
Background: Advanced technological aids are frequently used to improve outcome of transbronchial diagnostics for peripheral pulmonary lesions. Even when lesion access has been confirmed by 3D imaging, obtaining an accurate tissue sample however remains difficult. In this single institution study, we evaluate the comparative accuracy of different sampling methodologies and the accuracy of rapid on-site evaluation of cytopathology (ROSE) in navigation bronchoscopy cases where imaging has confirmed the catheter to have accurately accessed the lesion.

Methods: All consecutive navigation bronchoscopies in between December 2017-June 2020 performed in a room with a cone beam CT (CBCT) system where catheter position was intra-procedurally confirmed to be within or adjacent to the lesion by cone beam CT and augmented fluoroscopy were included. Individual tool outcomes were compared against one another and follow-up outcome.

Results: A mean of 11.39 samples using 2.93 tools were obtained in 225 lesions (median diameter $15 \mathrm{~mm}$, 195 patients). A correct diagnosis was most often obtained by forceps (accuracy $70.6 \%$ ), followed by $1.1 \mathrm{~mm}$ cryoprobe (68.4\%), needle aspiration (46.7\%), $1.9 \mathrm{~mm}$ cryoprobe (41.2\%), brush $(30.3 \%)$ and lavage $(23.7 \%)$. Procedural outcome corresponded to follow-up outcome in $75.1 \%$ of lesions ( $80.5 \%$ of patients). Accurately diagnosed lesions were sampled significantly more often (11.91 vs. 9.72 samples, $\mathrm{P}=0.014)$. In cases where procedural outcome proved malignant, ROSE had also detected this in $47.5 \%$.

Conclusions: Of all clinically available biopsy tools, the forceps showed most often accurate. However, extensive multi-modal sampling resulted in highest diagnostic accuracy. A hypothetical multi-modal approach of only using forceps and needle aspiration provided eventual diagnostic outcome in $91.7 \%$ of successfully diagnosed lesions. In the circumstances of our study, confirmation of malignancy on ROSE did not reduce number of biopsies taken nor biopsy time. Future research on how to improve the accuracy and effectivity of tissue sampling is needed.
\end{abstract}

Keywords: Biopsy; navigation bronchoscopy; peripheral pulmonary nodule; peripheral pulmonary lesion; rapid on-site evaluation of cytopathology

Submitted Mar 26, 2021. Accepted for publication Jun 23, 2021.

doi: $10.21037 /$ jtd-21-518

View this article at: https://dx.doi.org/10.21037/jtd-21-518

\footnotetext{
^ ORCID: Roel L. J. Verhoeven, 0000-0002-7892-4700; Shoko Vos, 0000-0001-7807-3056; Erik H. F. M. van der Heijden, 0000-0003$3596-518 X$.
} 


\section{Introduction}

Despite the development and introduction of several technological platforms to help guide the physician in the past two decades, obtaining an accurate endobronchial diagnosis of small peripheral pulmonary nodules remain a challenge. These technological platforms allow for both navigation guidance towards a lesion as well as confirming accurate lesion access, which become increasingly important as lesions are small sized and located peripherally in the lung. Techniques include ultrathin bronchoscopy, virtual navigation bronchoscopy, electromagnetic navigation, radial endobronchial ultrasound probes (rEBUS), robotic assisted bronchoscopy and cone beam CT imaging (CBCT) (1-10).

Unfortunately, a confirmed successful navigation does not warrant a representative and accurate diagnosis. Several studies report the diagnostic yield and procedural sensitivity being lower than that of navigation success $(2,10-12)$. Furthermore, a study by Coghlin et al. showed that the mean \% area of samples obtained from visible endobronchial lung cancer on histopathology was only $33.4 \%$ and additionally found that $52 \%$ of patients had one or multiple fragments collected which contained no tumor at all (13). As lesions become smaller and are of early stage, the margin of error and the nuance become smaller, and likely even less samples will reveal the true nature of the lesion.

Obtaining accurate tissue samples for analysis is made difficult due to small lesion size, tumor heterogeneity, and, the ability of making a clear distinction in (early state) disease pathology in samples of limited size. The concurrent decision-making process on which tissue sampling methodology to use and deciding on when sufficient material has been collected add to this complexity. Additional general characteristics influencing the likelihood of a diagnostic outcome are the positioning relative to the bronchus, lobes involved, solidness, malignancy presence, pleural distance and patient characteristics such as emphysema or other co-morbidity $(5,14-22)$. As two editorials recently concluded, there's however insufficient evidence to-in general-prefer one overall tissue acquisition method over the other, while several studies comparing individual technologies and concurrent methodologies have been published to date (2,23). Multiple studies suggest trans-bronchial needle aspiration (TBNA) has good outcome $(15,17,18)$, while the brush, forceps and cryobiopsy probes are other commonly available and successfully used means $(2,15,18,21,24-26)$. One further promising addition to the routine tissue sampling methodology is Rapid On-site Evaluation of cytopathology (ROSE), providing on-site information on cytology aspirate representativeness and the possibility of malignancy. It may enhance diagnostic yield and reduce complication rates in transbronchial and endobronchial sampling, although there is contradiction in results (27-29).

In this single center university hospital study, we comparatively assess the different tissue acquisition methodologies and the value of ROSE in a cohort of patients whom have been navigated to by CBCT guided navigation bronchoscopy for small peripheral pulmonary nodules. As the 3D CBCT image verification provides for tool analysis of only those lesions which have shown to be accurately accessed, it provides for a unique analysis. This article prospectively analyzes a cohort of consecutive patients in accordance with the STARD reporting checklist (available at https://dx.doi.org/10.21037/jtd-21-518). By relating biopsy tool outcomes per lesion, a direct paired comparison of tools is enabled whilst simultaneously incorporating clinical decision making on tool use. Combined, we report on overall diagnostic tool accuracy and agreement of ROSE with final pathology outcome in a navigation bronchoscopy setting.

\section{Methods}

\section{Patients}

This study was conducted in accordance with the Declaration of Helsinki (as revised in 2013) and took place in an experienced university hospital with a dedicated pulmonary pathology and interventional pulmonology team where navigation bronchoscopy is the first line diagnostic procedure for peripheral pulmonary lesions. Navigation bronchoscopy under 3D image guidance is only performed in those lesions where advanced navigation and/ or confirmation is deemed necessary. The need of advanced $3 \mathrm{D}$ imaging in addition to r-EBUS imaging or conventional C-arm fluoroscopy was based upon physician estimation of procedure difficulty taking into account lesion size and location ( $>2 \mathrm{nd}$ order branches beyond segmental bronchi or no bronchus sign). In this study, only cases subject to the CBCT guided navigation bronchoscopy were included where $3 \mathrm{D}$ confirmation of lesion access was available. All patients receiving a CBCT guided navigation bronchoscopy for diagnosis of a peripheral pulmonary lesion in the period of December 2017 to June 2020 were eligible and 


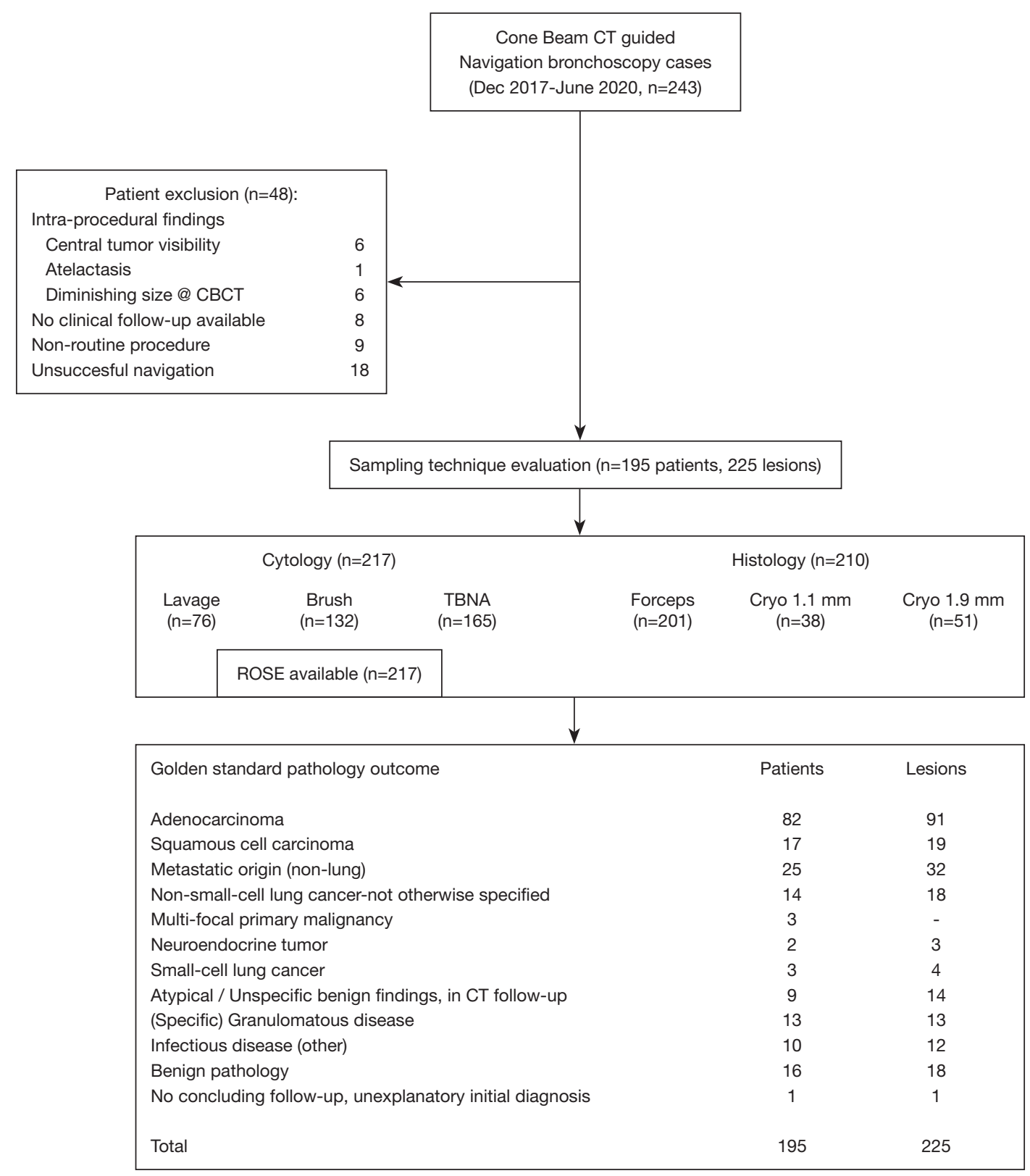

Figure 1 Flow diagram on study inclusion. Unsuccessful navigations were classified as those where imaging showed that the lesion was not successfully reached. A non-routine procedure indicated the primary reason for navigation bronchoscopy was other than diagnostics or different than normal procedural work-up due to study or clinical causes. ROSE was available only per analysis of brush and TBNA smears. TBNA, trans-bronchial needle aspiration; ROSE, rapid on-site evaluation of cytopathology.

prospectively approached for written informed study consent. Study approval was obtained by the independent local medical ethical committee (Arnhem-Nijmegen) and institutional review body (No: 2019-5148), informed consent was taken from all the patients. Patients in which a CBCT guided navigation setting was deemed unnecessary due to intra-procedural findings, or, patients in whom the lesion could not be successfully reached as verified by $3 \mathrm{D}$ CBCT, were excluded for analysis. After exclusion, only study subjects and concurrent peripheral lesions for which successful lesion access had been verified by means of navigation bronchoscopy remained for analysis (Figure 1). 


\section{Study method}

Conventional bronchoscopes with $2.8 \mathrm{~mm}$ working channel (EB19-J10, Pentax Medical, Japan) and consecutive $2.6 \mathrm{~mm}$ catheters (Olympus medical guide sheath, Tokyo, Japan \& Medtronic extended working channels, Minneapolis, USA) were used to navigate. Navigation was performed by one or a combination of electromagnetic navigation guidance (Medtronic SuperDimension), rEBUS (Olympus UMS20-17S) and CBCT imaging with augmented fluoroscopy (Philips Allura/Azurion, Best, The Netherlands or Siemens Zeego, Forcheim, Germany). The procedures took place under general anesthesia. After navigation, lesion access was verified by at least CBCT and augmented fluoroscopy, but rEBUS was also often additionally used.

After confirming navigation success, the catheter remained positioned near or within the lesion throughout biopsy specimen acquisition. Biopsy specimen acquisition was performed with intermittent augmented fluoroscopy, CBCT and/or rEBUS imaging. All tool use and the amount of sampling was decided upon by the endoscopy team while taking into account factors such as safety, hypothesized efficacy, needed diagnostic testing and lesion characteristics. The intermittent ROSE outcome of individual samples was used to decide upon the need of additional sampling. With the tools that were decided upon, biopsy specimen acquisition was routinely performed in the following order; brushing, TBNA, followed by forceps and/or cryobiopsy and lavage. Sampling was routinely started using brush or TBNA. Based on the CBCT and/or rEBUS imaging outcomes, we estimated which tool had highest chance of accurately accessing the target lesion and obtaining a sample. Typically, when a bronchus sign was identified and/ or rEBUS showed central access in a solid lesion, a brush was first obtained. In other cases, TBNA was the first tool of choice. Lavage was performed lastly and included injection of 10 to up to $60 \mathrm{~mL}$ of $0.9 \%$ saline through the catheter or endoscope. Subsequent $0.9 \%$ saline retrieval was attempted by suction until deemed sufficient for analysis. Brushing for cytopathology was performed by a pushing-pulling technique of the complete $1.8 \mathrm{~mm}$ brush in and out of the catheter (Olympus BC-202D-2010 brush, Medtronic SuperDimension cytology brush or Mediglobe cytology brush, Achenmühle, Germany). Needle aspiration was predominantly performed with $18 \mathrm{G}$ needles (Broncus FleXNeedle, San Jose, USA) by means of a similar motion as to that of the brush, with suction being applied by syringe proximally. Twenty-one gauge and 19G needles of other manufacturers were less often used, based upon commercial primary needle availability (Medtronic SuperDimension Aspirating Needle). Typically at least three aspirations were obtained with approximately ten strokes per aspiration. Serrated or non-serrated oval forceps of $1.8 \mathrm{~mm}$ diameter were used to acquire histology specimens (Boston Scientific Pediatric Radial Jaw 4 or Olympus FB233 D biopsy forceps). After advancement through the catheter, the forceps were opened and further pushed into the lumen until traction could be felt, after which they were closed and retracted for histology specimen collection. Cryobiopsy for histopathology was available only through a $1.9 \mathrm{~mm}$ cryoprobe until September 2019, after which a $1.1 \mathrm{~mm}$ version also became available (Erbe Elektromedizin, Tuebingen, Germany). Due to probe and sample size, the $1.9 \mathrm{~mm}$ cryoprobe had to be removed along with catheter and endoscope after an initial freeze of 4-7 s. Oppositely, the catheter could be left in place for repeated sampling with the $1.1 \mathrm{~mm}$ cryoprobe. Freezing during 5-9 s was followed by removal of the $1.1 \mathrm{~mm}$ probe for histopathology collection.

Individual brush and TBNA cytology samples were divided onto two slides; one Giemsa staining which was also used for ROSE and one slide for Papanicolaou smearing used only for definitive analysis. Remaining material was collected for cell block. Separate collection containers were used for every sampling tool. ROSE was available for all cases included in this study, which in our center is performed by a dedicated team of three experienced and trained cytopathology technicians who are routinely involved in all navigation bronchoscopy, EBUS, EUS and ultrasound guided TTNA procedures performed (totaling $>6,000$ procedures in the past decade). The ROSE procedure took place at the interventional pulmonology suite, such that twoway communication was easily maintained.

\section{Statistical analysis}

Descriptive parameters are presented as counts and percentages, along with medians, means and ranges. All individual tool outcomes and concurrent individual nodules were correlated to a procedural outcome and a golden standard follow-up outcome. Golden standard follow-up was either the final pathology diagnosis from surgical resection, additional CT guided TTNA and/or clinical follow-up with CT for at least 6 months was considered definitive. The comparative accuracy of tools was determined by pairing tool outcome in cases of individual lesions where two or more tools were used. The McNemar chi-squared test was 
Table 1 Patient, lesion and procedural characteristics

\begin{tabular}{lc}
\hline Variables & Number \\
\hline Patients/lesions (successfully accessed) & $195 / 225$ \\
Age, mean [range] (years) & $65.2[36-85]$ \\
Gender (M/F) & $103 / 92$ \\
Malignancy prevalence (pt./lesion) & $76 \% / 75 \%$ \\
Benign disease follow-up, median [range] & $491[215-1,224]$ \\
(days) & \\
Nodule size, median [range] (mm) & $15[5-65]$ \\
Bronchus sign (lesion) & $64.4 \%$ \\
Diagnostic accuracy procedure & $80.5 \%(157 / 195)$ \\
Diagnostic accuracy lesion & $75.1 \%(169 / 225)$ \\
Lesion locations & $62 / 77$ \\
LUL/RUL & 11 \\
RML & $32 / 43$ \\
LLL/RLL & \\
\hline
\end{tabular}

LUL, left upper lobe; RUL, right upper lobe; RML, right middle lobe; LLL, left lower lobe; RLL, right lower lobe.

used to test equivalence of tool accuracy. Wilcoxon testing was performed for comparison on not-normally distributed data. Student's $t$-tests were used to test accuracy differences between unpaired data. Tests with a $\mathrm{P}$ value of $<0.05$ were considered significant. For evaluation of ROSE, all procedures where ROSE was performed were included for analysis. The concordance of procedural malignant ROSE outcome by all cytological specimens was compared with both final procedural cytology as well as procedural histology outcome. R and RStudio were used for statistical analysis (30).

\section{Results}

A total of 195 patients received successful navigation bronchoscopy as verified by CBCT and augmented fluoroscopy imaging, in which a total of 225 lesions were shown to be successfully reached (Figure 1, Table 1). Malignant disease was found in $76 \%$ of patients. Median lesion diameter was $15.0 \mathrm{~mm}$ (range, 5-65 mm). Lesions were pure ground glass or part solid in $7.1 \%$ and $15.1 \%$ of cases, respectively. The procedural outcome (whilst navigation had showed successful) corresponded to gold standard outcome in $80.5 \%$ of cases on a per patient basis, and $75.1 \%$ of cases on a per lesion basis.

\section{Comparative tissue sampling method accuracy}

Analysis of individual tissue sampling methods revealed forceps biopsy was most often found accurate (70.6\%), followed by $1.1 \mathrm{~mm}$ cryoprobe findings (68.4\%), TBNA (46.7\%), $1.9 \mathrm{~mm}$ cryoprobe (41.2\%) and tiers brush (30.3\%) and lavage (23.7\%). The pair-wise tool comparison substantiated these accuracy findings (Figure 2). The forceps showed superior accuracy when directly compared against all cytology techniques (brush, TBNA, lavage $\mathrm{P}<0.01$ ), but was not significantly better than pooled 1.1 and $1.9 \mathrm{~mm}$ cryobiopsy accuracy $(\mathrm{P}=0.081)$. Pooled $1.1 \mathrm{~mm}$ and $1.9 \mathrm{~mm}$ cryobiopsy had significantly better accuracy than lavage and brush $(\mathrm{P}<0.05)$, but not TBNA $(\mathrm{P}=0.40)$. The $1.1 \mathrm{~mm}$ cryoprobe was more often accurate than the $1.9 \mathrm{~mm}$ cryoprobe (68.4\% vs. $41.2 \%$, respectively).

Analysis of a multi-modal sampling approach revealed sampling using forceps combined with TBNA would have provided eventual diagnostic outcome in 155 out of 169 successfully diagnosed lesions $(91.7 \%)$. In the cases where these were not diagnostic ( $\mathrm{n}=14)$, sampling by $1.1 \mathrm{~mm}$ and $1.9 \mathrm{~mm}$ cryoprobe correctly diagnosed 2 and 8 lesions whilst brushing and lavage were accurate in 4 and 3 lesions, respectively.

\section{Cytology versus histology}

The cytology findings (brush, TBNA, lavage) corresponded to gold standard follow-up outcome in $49.3 \%$ of lesions (107/217, Table 2). Histology findings (by forceps and cryobiopsy) were more often found accurate $(72.4 \%)$, but the combination of both cytology and histology remained most accurate $(75.1 \%)$. Analysis of data wherein both cytology and histology were obtained shows histology was significantly more often accurate, be it either malignant or benign (Table 2, P value <0.01). The histology outcome by itself was significantly more often accurate in benign lesions than in malignant lesions $(\mathrm{P}<0.01,88.6 \%$ vs. $66.9 \%$, respectively). This finding did not similarly hold for cytology outcome (48.5\% accuracy in benign lesions and $51.9 \%$ accuracy in malignant lesions, Table 2).

\section{Extensive multi-modal sampling}

Analysis of sampling method accuracy shows lesions whom were accurately diagnosed were subjected to a significantly higher amount of sampling (Table 3, $\mathrm{P}=0.014$ ). An average of 3.83 separate cytology samples (range, $0-15$ ) and 7.56 separate 


\begin{tabular}{|c|c|c|c|c|c|c|}
\hline & Lavage & Brush & TBNA & Forceps & Cryo $1.1 \mathrm{~mm}$ & Cryo 1.9 mm \\
\hline Lavage & $23.7 \%(18 / 76)$ & $\begin{array}{l}23.9 \% / 32.8 \% \\
(n=67, P=0.21)\end{array}$ & $\begin{array}{l}26.7 \% / 37.8 \% \\
(n=45, P=0.27)\end{array}$ & $\begin{array}{l}24.6 \% / 66.2 \% \\
(n=65, P<0.01)\end{array}$ & $\begin{array}{c}0 \% / 100 \% \\
(n=1, P=N A)\end{array}$ & $\begin{array}{c}0 \% / 46.6 \% \\
(n=15, P=N A)\end{array}$ \\
\hline Brush & & $30.3 \%(40 / 132)$ & $\begin{array}{l}21.0 \% / 43.2 \% \\
(n=81, P<0.01)\end{array}$ & $\begin{array}{c}31.1 \% / 66.9 \% \\
(n=119, P<0.01)\end{array}$ & $\begin{array}{c}20 \% / 90 \% \\
(n=10, P=0.023)\end{array}$ & $\begin{array}{l}25.8 \% / 38.7 \% \\
(n=31, P=0.34)\end{array}$ \\
\hline TBNA & & & $46.7 \%(77 / 165)$ & $\begin{array}{c}48.1 \% / 72.1 \% \\
(n=154, P<0.01)\end{array}$ & $\begin{array}{l}58.8 \% / 67.6 \% \\
(n=34, P=0.58)\end{array}$ & $\begin{array}{l}21.9 \% / 37.5 \% \\
(n=32, P=0.27)\end{array}$ \\
\hline Forceps & & & & $70.6 \%(142 / 201)$ & $\begin{array}{l}80.6 \% / 72.2 \% \\
(n=36, P=0.51)\end{array}$ & $\begin{array}{l}57.1 \% / 42.9 \% \\
(n=42, P=0.21)\end{array}$ \\
\hline Cryo $1.1 \mathrm{~mm}$ & & & & & $68.4 \%(26 / 38)$ & $\begin{array}{l}100 \% / 100 \% \\
(n=1, P=N A)\end{array}$ \\
\hline Cryo $1.9 \mathrm{~mm}$ & & & & & & $41.2 \%(21 / 51)$ \\
\hline
\end{tabular}

Figure 2 Sampling accuracy of different instruments as used in the navigation bronchoscopy procedure. Diagonal (grey boxes): accuracy of the individual instrument, as calculated over the amount of times the instrument was used (n/N). Top right half of table: Pair-wise comparison of tool accuracy, calculated by evaluation of cases where both tools were used in the same lesion. The individual tool accuracies as calculated by every time they were pair-wise used are shown in red (rows) and blue (columns). I.e. the first row shows how often lavage was accurate versus how often other tools were accurate (in red), when both were used (in blue). In between brackets (in black) the number of times the pair-wise tool comparison was available (as both tools were used in a single lesion), followed by probability of significant accuracy differences between the two tools. NA, not applicable; TBNA, trans-bronchial needle aspiration; Cryo, cryoprobe sampling; n, amount of cases; p, probability outcome of McNemar chi-squared test for pair-wise comparison of instruments.

histology samples (range, 0-18) were acquired per procedure, equivaling an overall average of 11.39 samples (range, 1-25). In case they were used, collection of samples by forceps and $1.1 \mathrm{~mm}$ cryobiopsy was performed an average of 7.08 and 6.12 times per lesion, respectively. Brush and TBNA-both enabling ROSE - were used for repeat sampling a respective average of 1.64 and 3.26 times (Table 3). Overall outcome further showed that the forceps and TBNA sampling tools were used most of all tools, respectively having been used in $89.3 \%$ and $73.3 \%$ of lesions (Figure 2 and Table 3).

\section{Rapid on-site evaluation of cytopathology}

Rapid on-site evaluation of cytopathology was available in cases where sampling by brush and/or TBNA was performed $(\mathrm{n}=217)$. Of 79 lesions where cytology analysis was suggestive of malignancy, ROSE concluded similarly in 57 (69.8\%). Due to the discrepancy between cytology results and overall pathology results, ROSE was able to conclude malignancy in $47.5 \%$ of cases where procedural outcome showed malignancy (57/120, Table 2). In the procedures where ROSE was found suggestive of malignancy, significantly more samples were obtained than where it did not (respective average of 12.65 vs. 10.51 samples, $\mathrm{P}=0.016$, Table 3). The biopsy time insignificantly correlated to these findings, taking an average of 25.1 minutes in ROSE findings suggestive of malignancy and 24.2 minutes in non-confirmatory ROSE findings $(\mathrm{P}=0.36)$.

\section{Discussion}

In navigation bronchoscopies where access of lesions with a mean $15 \mathrm{~mm}$ size had been verified through (repeated) $3 \mathrm{D}$-imaging, we found that procedural pathology outcome corresponded to follow-outcome in $80.5 \%$ per patient $(75.1 \%$ of lesions). An accurate diagnosis was most often obtained by forceps (accuracy 70.6\%), followed by $1.1 \mathrm{~mm}$ cryoprobe (68.4\%), TBNA (46.7\%), $1.9 \mathrm{~mm}$ cryoprobe (41.2\%), brush $(30.3 \%)$ and lavage $(23.7 \%)$. Our pair-wise comparison of tools showed a similar order of individual tool yield. By these findings, histology results alone were found representative of follow-up outcome in $72.4 \%$, and by cytology in $49.3 \%$ of cases (Figure 2 and Table 3, P value $<0.01$ ). Due to the discordance of cytology findings with overall procedural findings, and ROSE again being discordant with cytology (in $27.9 \%$ of cases), ROSE was able to help predict procedural malignant pathology outcome in only $47.5 \%$ of cases.

During the conduct of this study, (intermittent) 3D-imaging verification was frequently used both after navigation and in-between repeated sampling. We 
Table 2 Sampling outcome (per lesion) versus procedural and follow-up gold standard outcome

\begin{tabular}{|c|c|c|}
\hline Pathology outcome-per lesion basis & $\%$ & $\mathrm{n}$ \\
\hline ROSE correlating to malignant cytopathology findings & $72.1 \%$ & $57 / 79$ \\
\hline ROSE correlating to procedural malignant findings (cytology \& histology combined) & $47.5 \%$ & $57 / 120$ \\
\hline \multicolumn{3}{|l|}{ Sampling accuracy in malignant lesions } \\
\hline Histopathology (procedural) accuracy & $66.9 \% *, \star \star$ & $105 / 157$ \\
\hline Combined pathology (procedural) accuracy & $71.0 \%$ & $120 / 169$ \\
\hline Molecular analysis possible ${ }^{\dagger}$ & $83.9 \%$ & $26 / 31$ \\
\hline \multicolumn{3}{|l|}{ Sampling accuracy in benign lesions } \\
\hline Combined pathology (procedural) accuracy & $87.5 \%$ & $49 / 56$ \\
\hline \multicolumn{3}{|l|}{ Sampling accuracy-lesions overall } \\
\hline Cytopathology (procedural) accuracy & $49.3 \% *$ & $107 / 217$ \\
\hline Histopathology (procedural) accuracy & $72.4 \% *$ & $152 / 210$ \\
\hline Overall pathology (procedural) accuracy & $75.1 \%$ & $169 / 225$ \\
\hline
\end{tabular}

Results of rapid on-site evaluation (ROSE) of cytopathology are described specifically for malignant disease findings as found per procedural cytopathology outcomes and overall procedural outcomes (being malignant). As clinical decision making decided on tool use, only cases where cytology or histology was available were used for calculation of accuracy. *, significant differences (P value $<0.01$, McNemar chi-squared test) between accuracies of cytology and histology in benign, malignant and lesions overall were found when assessing paired outcomes. ${ }^{* *}$, significant difference ( $P$ value $<0.01$, unpaired two-sided Student's $t$-test) between accuracy of histology outcomes in benign and malignant lesions. ${ }^{\dagger}$, molecular analysis is only performed upon request (when clinically indicated), denoted here is the number of times it was possible when requested.

hypothesize the use of imaging in combination with maintained catheter position were essential in meticulous positioning and acquiring tissue at different sites of hypothesized pathology. It is likely only a minority of tissue samples contains pathologic tissue, as also indicated by Coghlin et al. in biopsy samples of visible lung cancers (13). The NAVIGATE study and a recent systematic review and meta-analysis substantiate these findings, finding a higher procedural success in cases of multi-modal and extensive sampling $(12,31)$. We also found significantly more sampling was performed in lesions where an accurate diagnosis could be obtained (9.72 vs. 11.91 samples, $\mathrm{P}=0.014$ ). Our repeated sampling with tools was based on clinical decision making rather than being a randomized and controlled trial. With the available tools having different properties, we deemed it relevant to tailor their use to the situation. In general, we chose our tools based upon relative positioning of the catheter to the lesion and secondary adjacent structures (e.g., pleura, vessels, cavities). When a central position in the lesion was obtained, a brush was generally the first choice (followed by secondary sampling tools). When lesion position was eccentric or trans-parenchymal access was deemed necessary, TBNA was often preferred first. The choice to not perform repeated TBNA, brush or lavage sampling more often was made consciously. EBUS-TBNA and ROSE literature has shown acquisition of more than 4 TBNA samples in EBUS is of minimal additional value and sufficient for enabling molecular analysis in the vast majority of cases (32). While we cannot perform sampling under direct ultrasonic or video guidance, the motion and straightening of the catheter after needle insertion along with ROSE findings often being consistent in outcome from first to last biopsy made us decide on reducing routine TBNA use to 3-4 times. The choice of forceps and higher 
Table 3 Descriptive statistics on procedural sampling techniques

\begin{tabular}{lcccc}
\hline Descriptive statistics on sampling techniques & Mean (samples) & Median (samples) & Min-max (samples) & Accuracy (tool) \\
\hline Cytology & 3.83 & 4 & $1-15$ & $107 / 217(49.3 \%)$ \\
Lavage & 1.18 & 1 & $1-3$ & $18 / 76(23.7 \%)$ \\
Brush & 1.64 & 2 & $1-4$ & $40 / 132(30.3 \%)$ \\
TBNA & 3.26 & 3 & $1-11$ & $77 / 165(46.7 \%)$ \\
Histology & 7.56 & 7 & $0-18$ & $152 / 210(72.4 \%)$ \\
Forceps & 7.08 & 7 & $1-18$ & $142 / 201(70.6 \%)$ \\
Cryobiopsy $(1.1 \mathrm{~mm})$ & 6.12 & 7 & $1-10$ & $26 / 38(68.4 \%)$ \\
Cryobiopsy $(1.9 \mathrm{~mm})$ & 1.15 & 1 & $1-2$ & $21 / 51(41.2 \%)$ \\
Samples total & 11.39 & 11 & $1-25$ & $169 / 225(75.1 \%)$ \\
ROSE: malignant findings & $12.65^{\dagger}$ & 12 & $4-24$ & - \\
ROSE: benign findings & $10.51^{\dagger}$ & 10 & $1-25$ & - \\
Unsuccessful diagnosis & $9.72^{\ddagger}$ & 10 & $1-23$ & - \\
Successful diagnosis & $11.91^{\ddagger}$ & 12 & $1-25$ & - \\
Tools total & 2.93 & 3 & $1-6$ & - \\
\hline
\end{tabular}

Amount of sampling obtained are computed from cases where the instrument was used to acquire at least one sample. The accuracy are furthermore given by comparing the amount of times the instrument was used and led to an accurate diagnosis as compared against the total times it was used. On the bottom half of the table, the total amount of samples obtained for the different types of procedure characteristics are furthermore given; amount of samples when ROSE concluded malignancy, did not conclude malignancy, amount of samples in undiagnostic lesions and in cases where an accurate diagnosis could be obtained. ${ }^{\dagger}, \mathrm{P}$ value $=0.016$, significantly more samples were obtained when ROSE had concluded malignancy; ${ }^{\ddagger}, \mathrm{P}$ value $=0.014$, significantly more samples were obtained in accurate procedures. TBNA, trans-bronchial needle aspiration; ROSE, rapid on-site evaluation of cytopathology.

amounts of repeat biopsy when compared to other tools was partially based on the observation that precurved catheters as used in this study lost least of their curvature by forceps, consequently allowing for biopsy of different sites. This could also provide for an explanation why we find a significant cytology and histology accuracy difference in both diagnosing benign and malignant lesions (Table 2). But where cytology accuracy was similar in malignant and benign lesions (48.5\% vs. 51.9\%, respectively), histology outcome was of higher variation $(66.9 \%$ vs. $88.6 \%$ for malignant and benign outcomes, respectively). We cannot clearly explain this histology accuracy difference in benign and malignant findings, as it is also contrary to generalized findings of other studies (31). As such, additional research remains needed.

Procedural ROSE is likely of highest added value if feedback and communication between the interventional pulmonology and ROSE team is continuously had and a standardization of the process is agreed upon. As previously found, the ROSE methodology may however differ among centers, with no evidence for preferring one above another smearing method (32). A meta-analysis by Mondoni et al. found ROSE increases the procedural yield also in diagnosis of peripheral lesions (15). Moreover, previous reports have shown that molecular analysis in EBUS-TBNA can be performed more often in a ROSE enhanced procedure (29). In this study, we uniquely use Cone beam CT imaging to verify in $3 \mathrm{D}$ that the lesion has been accurately accessed. Consecutively, extensive sampling was performed. Yet even after having (repeatedly) verified lesion access, a diagnosis indicative of procedural malignant outcome could be obtained by ROSE in only $47.5 \%$ of cases (Table 2). The discordant findings when related to procedural and cytology outcome may in part be explained by differences in available material. ROSE provides outcome on Giemsa staining, whilst additional material is harbored within Papanicolaou staining and cell block. Another cause of discordance might be related to the cytopathologist's opinion that analysis of peripheral pulmonary lesion smears is different to that of EBUS-TBNA, being more diverse. 
With decreasing lesion size and less solidity, differentiating between atypical findings and malignancy becomes more difficult (2). Combined, we can conclude that ROSE was of additional value in less than $50 \%$ of cases, and, that it was not associated with a reduction of total number of samples taken nor with biopsy time. However, having ROSE, the feedback obtained from our team enabled us to adapt our sampling strategy per nodule. The results of our study propose that more sampling is performed in cases where ROSE is confirmatory of malignancy. This is opposite to one would expect, as it should logically allow for earlier abandoning of additional sampling instead. With high chance of malignancy, we wanted to be sure to have sufficient tissue for a complete analysis in the modern era. Therein, even when we had a clear confirmation on the presence of malignancy and confirmation that the sample contained sufficient cells (or estimated DNA) for full analysis, we likely still obtained additional histology samples to maximize the chance of confirmatory outcome for the patient and avoid the possibility of needing re-biopsy. As a result, in these circumstances, having ROSE to confirm malignancy did not translate into less samples taken.

To our knowledge, this is one of first clinical trials to report on $1.1 \mathrm{~mm}$ cryoprobes for peripheral lesion biopsy in a through-the-catheter approach. Our initial experience with the $1.9 \mathrm{~mm}$ cryoprobe was cumbersome, as it often dislocated initial catheter position upon insertion due to probe rigidity. This especially was problematic in cases with tight instrument angulations, and could not be compensated for completely in several cases. Consequently, we feel the probe was often not as optimally positioned when compared to other instruments. What's more, due to the probe's size, the probe had to be removed together with the catheter for a single specimen removal. As a result, only one sample could be obtained at the end of the procedure. The newer $1,1 \mathrm{~mm}$ cryoprobe allows for repeated sampling through the extended working channel, is much less rigid and as such; easier to use. As descriptively reported, a combination of TBNA with forceps biopsy in a curved guide sheath however deprecated the added value of also adding this tool to the inventory in 22 out of 24 cases where it was found diagnostic. It has previously been described that especially lesions nonconcentrically positioned around the bronchus would be benefitted by the cryoprobe (21). Our findings show this might be less valid for the smaller sized probe, which could be caused by its difference in area and tissue penetration.

Folch $e t a l$. and the NAVIGATE trial report a higher degree of sampling and multi-modal sampling led to higher yield $(12,31)$. We report routinely sampling more than 10 times per lesion in a multi-modal fashion and similarly see a higher amount of sampling correlating to finding an accurate diagnosis by procedural pathology more often (Table 3, $\mathrm{P}=0.014)$. Our high degree of repeat sampling was in part based on our clinical findings that lesion access by imaging verification was frequent, but a confirmatory diagnosis could then not always be made. The hypothetic TBNA and forceps only scenario as presented in this study however shows we would have found $91.7 \%$ of eventual diagnoses by only using these two modalities. It could be suggested that not all lesions require extensive multi-modal sampling. Yet, it can also be concluded that no commercially and readily available tool is currently a do-it-all tool even if lesion access has been confirmed. Therefore, multi-modal and repeated sampling seems to remain the recommended methodology.

\section{Conclusions}

This study evaluates the outcome of different tissue sampling tools and ROSE in a subset of navigation bronchoscopies where $3 \mathrm{D}$-imaging had verified lesion access in a routine clinical setting using widely available sampling tools. With repeated multimodal sampling, pathology outcome was found corresponding to gold standard follow-up outcome in $75.1 \%$ of lesions, resulting in $80.5 \%$ of patients obtaining a representative diagnosis. In lesions where an accurate diagnosis could be obtained, significantly more sampling was performed (9.72 vs. 11.91 samples, $\mathrm{P}=0.014$ ). An accurate diagnosis was most often obtained by forceps (accuracy $70.6 \%$ ), followed by $1.1 \mathrm{~mm}$ cryoprobe (68.4\%), TBNA (46.7\%), $1.9 \mathrm{~mm}$ cryoprobe (41.2\%), brush (30.3\%) and lavage $(23.7 \%)$. Analysis of a multi-modal sampling approach reveals sampling using only forceps and TBNA would have provided eventual diagnostic outcome in $91.7 \%$ of successfully diagnosed lesions. In cases where procedural pathology outcome proved malignant, ROSE had precedingly confirmed malignancy in $47.5 \%$. Confirmation of malignancy from ROSE during these procedures did not translate into a reduction of the number of biopsies taken or biopsy time. In conclusion, there is currently no single tool or methodology that is a do-it-all. Future research on how to improve the accuracy and effectivity of tissue sampling in navigation bronchoscopy is needed.

\section{Acknowledgments}

Funding: This work or part of this work was supported by 
unrestricted research grants from Philips, the Ankie Hak fund, Astra Zeneca Oncology Netherlands and in-kind support of Pentax Medical Europe.

\section{Footnote}

Reporting Checklist: The authors have completed the STARD reporting checklist. Available at https://dx.doi. org/10.21037/jtd-21-518

Data Sharing Statement: https://dx.doi.org/10.21037/jtd-21-518

Conflicts of Interest: All authors have completed the ICMJE uniform disclosure form (available at https://dx.doi. org/10.21037/jtd-21-518). RLJV reports grants and nonfinancial support from Philips, personal fees and nonfinancial support from Medtronic, Pentax Medical Europe, during the conduct of the study; grants from AstraZeneca Oncology, grants from the Ankie Hak Fund, grants from Siemens Healthineers outside the submitted work. Erik HFMH reports grants from Philips, personal fees and nonfinancial support from Medtronic and Pentax Medical Europe, during the conduct of the study; grants from AstraZeneca Oncology, grants from the Ankie Hak Fund, grants from Pentax Medical Europe and personal fees from Cook Medical, outside the submitted work. SV has no conflicts of interest to declare.

Ethical Statement: The authors are accountable for all aspects of the work in ensuring that questions related to the accuracy or integrity of any part of the work are appropriately investigated and resolved. The study was conducted in accordance with the Declaration of Helsinki (as revised in 2013). The study protocol was approved by the independent local medical ethical committee (ArnhemNijmegen) and institutional review body before start of subject inclusion (file number 2019-5148). Informed written consent was obtained from all study subjects.

Open Access Statement: This is an Open Access article distributed in accordance with the Creative Commons Attribution-NonCommercial-NoDerivs 4.0 International License (CC BY-NC-ND 4.0), which permits the noncommercial replication and distribution of the article with the strict proviso that no changes or edits are made and the original work is properly cited (including links to both the formal publication through the relevant DOI and the license). See: https://creativecommons.org/licenses/by-nc-nd/4.0/.

\section{References}

1. Wang Memoli JS, Nietert PJ, Silvestri GA. Metaanalysis of guided bronchoscopy for the evaluation of the pulmonary nodule. Chest 2012;142:385-93.

2. Fielding D, Oki M. Technologies for targeting the peripheral pulmonary nodule including robotics. Respirology 2020;25:914-23.

3. Oki M, Saka H, Asano F, et al. Use of an Ultrathin vs Thin Bronchoscope for Peripheral Pulmonary Lesions: A Randomized Trial. Chest 2019;156:954-64.

4. Callister ME, Baldwin DR, Akram AR, et al. British Thoracic Society guidelines for the investigation and management of pulmonary nodules. Thorax 2015;70 Suppl 2:ii1-ii54.

5. Rivera MP, Mehta AC, Wahidi MM. Establishing the diagnosis of lung cancer: Diagnosis and management of lung cancer, 3rd ed: American College of Chest Physicians evidence-based clinical practice guidelines. Chest 2013;143:e142S-e165S.

6. Fielding DIK, Bashirzadeh F, Son JH, et al. First Human Use of a New Robotic-Assisted Fiber Optic Sensing Navigation System for Small Peripheral Pulmonary Nodules. Respiration 2019;98:142-50.

7. Yarmus L, Akulian J, Wahidi M, et al. A Prospective Randomized Comparative Study of Three Guided Bronchoscopic Approaches for Investigating Pulmonary Nodules: The PRECISION-1 Study. Chest 2020;157:694-701.

8. Ali EAA, Takizawa H, Kawakita N, et al. Transbronchial Biopsy Using an Ultrathin Bronchoscope Guided by Cone-Beam Computed Tomography and Virtual Bronchoscopic Navigation in the Diagnosis of Pulmonary Nodules. Respiration 2019;98:321-8.

9. Pritchett MA, Schampaert S, de Groot JAH, et al. ConeBeam CT With Augmented Fluoroscopy Combined With Electromagnetic Navigation Bronchoscopy for Biopsy of Pulmonary Nodules. J Bronchology Interv Pulmonol 2018;25:274-82.

10. Verhoeven RLJ, Fütterer JJ, Hoefsloot W, et al. Cone-Beam CT Image Guidance With and Without Electromagnetic Navigation Bronchoscopy for Biopsy of Peripheral Pulmonary Lesions. J Bronchology Interv Pulmonol 2021;28:60-9.

11. Hohenforst-Schmidt W, Zarogoulidis P, Vogl T, et al. Cone Beam Computertomography (CBCT) in Interventional Chest Medicine - High Feasibility for Endobronchial Realtime Navigation. J Cancer 2014;5:231-41. 
12. Gildea TR, Folch EE, Khandhar SJ, et al. The Impact of Biopsy Tool Choice and Rapid On-Site Evaluation on Diagnostic Accuracy for Malignant Lesions in the Prospective: Multicenter NAVIGATE Study. J Bronchology Interv Pulmonol 2021;28:174-83.

13. Coghlin CL, Smith LJ, Bakar S, et al. Quantitative analysis of tumor in bronchial biopsy specimens. J Thorac Oncol 2010;5:448-52.

14. Ali MS, Sethi J, Taneja A, et al. Computed Tomography Bronchus Sign and the Diagnostic Yield of Guided Bronchoscopy for Peripheral Pulmonary Lesions. A Systematic Review and Meta-Analysis. Ann Am Thorac Soc 2018;15:978-87.

15. Mondoni M, Sotgiu G, Bonifazi M, et al. Transbronchial needle aspiration in peripheral pulmonary lesions: a systematic review and meta-analysis. Eur Respir J 2016;48:196-204.

16. Gould MK, Donington J, Lynch WR, et al. Evaluation of individuals with pulmonary nodules: when is it lung cancer? Diagnosis and management of lung cancer, 3rd ed: American College of Chest Physicians evidence-based clinical practice guidelines. Chest 2013;143:e93S-e120S.

17. Ost DE, Ernst A, Lei X, et al. Diagnostic Yield and Complications of Bronchoscopy for Peripheral Lung Lesions. Results of the AQuIRE Registry. Am J Respir Crit Care Med 2016;193:68-77.

18. Trisolini R, Cancellieri A, Tinelli C, et al. Performance characteristics and predictors of yield from transbronchial needle aspiration in the diagnosis of peripheral pulmonary lesions. Respirology 2011;16:1144-9.

19. Choi SH, Chae EJ, Kim JE, et al. Percutaneous CTguided aspiration and core biopsy of pulmonary nodules smaller than $1 \mathrm{~cm}$ : analysis of outcomes of 305 procedures from a tertiary referral center. AJR Am J Roentgenol 2013;201:964-70.

20. De Filippo M, Saba L, Concari G, et al. Predictive factors of diagnostic accuracy of CT-guided transthoracic fineneedle aspiration for solid noncalcified, subsolid and mixed pulmonary nodules. Radiol Med 2013;118:1071-81.

21. Kho SS, Chan SK, Yong MC, et al. Performance of transbronchial cryobiopsy in eccentrically and adjacently orientated radial endobronchial ultrasound lesions. ERJ Open Res 2019;5:00135-2019.

22. Nasu S, Okamoto N, Suzuki H, et al. Comparison of the Utilities of Cryobiopsy and Forceps Biopsy for Peripheral Lung Cancer. Anticancer Res 2019;39:5683-8.

23. Mondoni M, Sotgiu G. Bronchoscopic management of peripheral pulmonary lesions: robotic approach paves the way to the future. BMC Pulm Med 2019;19:166.

24. Chao TY, Chien MT, Lie CH, et al. Endobronchial ultrasonography-guided transbronchial needle aspiration increases the diagnostic yield of peripheral pulmonary lesions: a randomized trial. Chest 2009;136:229-36.

25. Herath S, Yap E. Novel hybrid cryo-radial method: an emerging alternative to CT-guided biopsy in suspected lung cancer. A prospective case series and description of technique. Respirol Case Rep 2018;6:e00287.

26. Schuhmann M, Bostanci K, Bugalho A, et al. Endobronchial ultrasound-guided cryobiopsies in peripheral pulmonary lesions: a feasibility study. Eur Respir J 2014;43:233-9.

27. Chen X, Wan B, Xu Y, et al. Efficacy of rapid onsite evaluation for diagnosing pulmonary lesions and mediastinal lymph nodes: a systematic review and metaanalysis. Transl Lung Cancer Res 2019;8:1029-44.

28. Mondoni M, Carlucci P, Di Marco F, et al. Rapid onsite evaluation improves needle aspiration sensitivity in the diagnosis of central lung cancers: a randomized trial. Respiration 2013;86:52-8.

29. Trisolini R, Cancellieri A, Tinelli C, et al. Randomized Trial of Endobronchial Ultrasound-Guided Transbronchial Needle Aspiration With and Without Rapid Onsite Evaluation for Lung Cancer Genotyping. Chest 2015;148:1430-7.

30. R Core Team. R: A language and environment for statistical computing. Vienna, Austria.: R Foundation for Statistical Computing; 2019.

31. Folch EE, Labarca G, Ospina-Delgado D, et al. Sensitivity and Safety of Electromagnetic Navigation Bronchoscopy for Lung Cancer Diagnosis: Systematic Review and Metaanalysis. Chest 2020;158:1753-69.

32. van der Heijden EH, Casal RF, Trisolini R, et al. Guideline for the acquisition and preparation of conventional and endobronchial ultrasound-guided transbronchial needle aspiration specimens for the diagnosis and molecular testing of patients with known or suspected lung cancer. Respiration 2014;88:500-17.

Cite this article as: Verhoeven RLJ, Vos S, van der Heijden EHFM. Multi-modal tissue sampling in cone beam CT guided navigation bronchoscopy: comparative accuracy of different sampling tools and rapid on-site evaluation of cytopathology. J Thorac Dis 2021;13(7):4396-4406. doi: 10.21037/jtd-21-518 